\title{
Ambulatory Monitoring of Maternal and Fetal using Deep Convolution Generative Adversarial Network for Smart Health Care IoT System
}

\author{
S. Venkatasubramanian \\ Department of Computer Science \\ Saranathan College of Engineering, Trichy-620012, India
}

\begin{abstract}
With the increase in the number of high-risk pregnancies, it is important to monitor the health of the fetus during pregnancy. Major advances in the field of study have led to the development of intelligent automation systems that enable clinicians to predict and determine the monitoring of Maternal and Fetal Health (MFH) with the aid of the Internet of Things (IoT). This paper provides a solution for monitoring high-risk MHF based on IoT sensors, data analysis-based feature extraction, and an intelligent system based on the Deep Convolutional Generative Adversarial Network (DCGAN) classifier. Various clinical indicators such as heart rate of MF, oxygen saturation, blood pressure, and uterine tonus of maternal are monitored continuously. Many data sources produce large amounts of data in different formats and ratios. The smart health analytics system proposes to extract several features and measure linear and non-linear dimensions. Finally, a DCGAN has been proposed as a predictive mechanism for the simultaneous classification of MFH status by considering more than four possible outcomes. The results showed that the proposed system for mobile monitoring between MFH is a practical solution based on the IoT.
\end{abstract}

Keywords-Deep convolutional generative adversarial network; fetal health monitoring; high-risk pregnancies; internet of things; smart healthcare system

\section{INTRODUCTION}

Remote monitoring systems in the healthcare domain are increasing the daily reach of health for at-risk populations, especially pregnant women [1] and the elderly [2]. To identify the early disease symptoms and provide care, the patients are monitored every second by using these promising techniques in healthcare. The major functionality of the system is to diagnose and predict the health conditions of the user and provide warnings and training for the same. Nowadays, recent advances in the technologies of IoT have presented a way to enable such monitoring services $24 / 7$. The IoT is a growing network of interconnected objects that include shared knowledge about decision-making and efficient and autonomous operation [3-6]. Various sources such as computer knowledge, communication link, and sensitivity are used by IoT in healthcare. As the content of public health, MFH is highly regarded by governments. However, medical care services, especially obstetric care, are limited, which decreases the efficiency of the medical staff and increased the pressure of high-quality service.
Although the fetus is located inside the body of a pregnant woman, the most devoted protector of the fetus is only the pregnant woman. Without the help of external technology, a mother will not be able to know the FH even when it is lifethreatening. Therefore, to study the $\mathrm{MH}$, monitoring the fetal is an important tool [7]. Fetal monitoring is reliable, safe, and easy to operate, which is widely practiced by $\mathrm{MCH}$ organizations across India [8-10]. However, the fetal monitoring used by most hospitals still traces deficiencies. First, the information cannot be shared. Monitor results must be printed in most hospitals, making them unsuitable for storage and easy to lose, and sharing tracking information and advice from multiple individuals may not feel timely.

These factors make it difficult for doctors to perform medical tasks or delay illness, which can lead to medical accidents. When pregnant women are in the obstetrics department, early warning function and intelligent real-time monitoring system are unable and they face other issues such as high risk, high emergencies, and massive flow of other pregnant women. In addition, serious consequences and lifethreatening risks have occurred, when pregnant women are unable to notify doctors in time. Finally, monitoring of human health at home fails to recognize: at present, traditional health systems cannot meet the needs of continuous monitoring of pregnant women. Pregnant women, as expectant mothers, play an indispensable role in the upbringing of their children. Therefore, self-monitoring at home is an important content during the period of perinatal health care.

In recent years, communities and families are greatly entering into the comprehensive application of information platforms such as cloud computing technology, IoT healthcare, big data, communication technology, etc. It provides individuals with purposeful and personalized services, enabling them to advance from telemedicine to new ideas and ways of preventing disease and addressing major public health issues in the lives of women and children [1113]. The IoT [14] means things are connected via the Internet that, understands the communication between objects using modern information technologies such as smart sensing, identification technology, and wireless communication. IoTbased M-Health uses wearable clinical sensors [15-17] to provide patients with real-time feedback on key symptoms and medical information and provide them with "anytime, anywhere" healthcare. 
Healthcare as a Service platform architecture for cloudbased medical decision support services is developed in [18]. In [19], a coherent framework for M-Health monitoring and IoT-based remote monitoring is proposed. In [20], the IoTbased cloud service is optimized for the next generation of smart environments. In wireless heterogeneous networks, architecture of hierarchical sensor-based healthcare is developed by the work [21]. Other work [22] focused on a variety of wireless access networks and provided a framework for remote patient monitoring services. In some other cases, the software is developed by the IoT infrastructure for safe and smart healthcare [23].

An example of GAN model architecture is shown as below in Fig. 1.

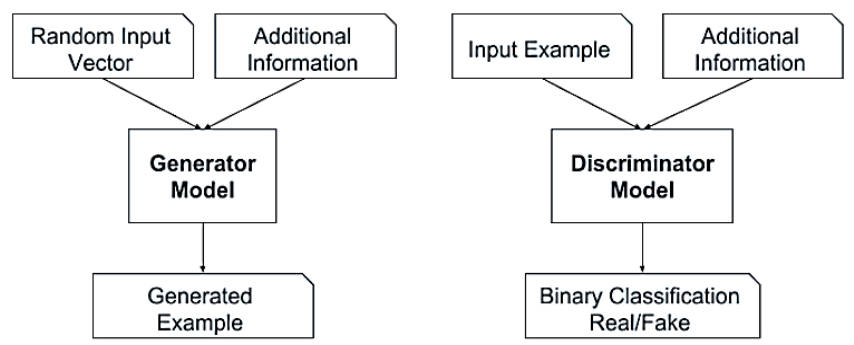

Fig. 1. Example of Generative Adversarial Network Model Architecture.

An integrated solution is proposed in this work for collecting different data from devices and sensors of IoT, linear and non-linear features are extracted, emergency alarms are used to analyze the data and finally, the MFH is automatically predicted by the DCGAN model. The most significant contributions are presented here as:

1) A continuous monitoring of MFH is carried out by integrating the medical devices and sensors of IoT.

2) To improve the diagnostic accuracy, fog computing architecture is used to develop an automated emergency subsystem with inference rules set.

3) The extraction of linear and non-linear features are done by a smart health analytics system.

4) The status of MFH is classified by analyzing the multiple metrics with the help of the DCGAN model.

This research paper is structured as follows. The related works for monitoring the fetal and maternal status are given in Section 2. A brief explanation of the system model with a predictive model is presented in Section 3. The validation of existing techniques with the proposed algorithm is described in Section 4. Finally, the scientific contribution of the research work with its future development is depicted in Section 5.

\section{RELATED WORK}

A safe and reliable monitoring framework at less costeffective is developed by Allah et al., [24] to make the home more comfortable and mitigate the effects of preterm labor in pregnant women. Non-invasive technology is used by the system to monitor EHG defects using wireless body sensors and smartphones. The smartphone will check for contractions and alert you in case of early delivery. Using the cervical contractility database, this smartphone app has been developed and tested for verifying reliability and performance in terms of power consumption. The analysis showed that this application serves the purposes of the framework in defining the work system.

The significance of variants with similar features between the EPL models and the current pregnancy models is proposed by Liu, et al., [25]. After the collection of embryo samples, the correlation between the heart rate of the fetus is identified, where a regression model is used to achieve the normal development of FHR. The remaining analysis reveals the importance of the FHR in determining pregnancy outcomes. To develop the computational models, this paper developed six different machine learning (ML) techniques are developed. Sensitivity is used to compare the accuracy of both the presence of FHR and conditions of absence for predicting the performance. FHR is closely related to ETD for normal development and attained a high-performance value. When compared with all techniques, the random forest has $97 \%$ of recall, F1-score, and accuracy with 0.97 of AUC; however, deep learning techniques are required for effective prediction.

During pregnancy, the exposure of incarceration and food insecurity is identified by Testa et al., [26]. From 2004 to 2015, the LR was used to determine the relationship between father and mother of a fetus' food insecurity using Pregnancy Risk Assessment System (PRAMS) results. Withdrawal of controls is associated, directly or indirectly, with a $165 \%$ increase in the risk of food instability. Attendance analysis indicates that this relationship is driven by various factors such as receiving WIC benefits, unemployment of maternal and financial hardship.

Azimi et al., [27], established a flexible decision-making mechanism to provide 24/7 health outcomes in the absence of data, despite missing policies. Various data resources are leveraged in IoT systems for providing the results and imputing the mission values. This approach was validated in the Human Maternal Health Cycle Research, where 20 pregnancies were observed for 7 months. The health status of the maternal is measured by using her heart rate in real-time applications. While comparing with existing related works, the accuracy is highly achieved by this developed method. But, the accuracy results are low, when there are large missing window size data presents.

The website is developed by Mourad $\mathrm{M}$ et al., [28], and the signalling site is used to study uterine contractions, their characteristics, and labour study. In this investigation, different nonlinear techniques are used to determine pregnancy and labor signals and to examine pregnancy signals before labor with Hogworth frontiers, the multifaceted nature of Lempel-Ziv, and the measurement of a fractal. In Lebanon and France, all data from $12 \mathrm{WBL}$ to $1 \mathrm{WBL}$ are recorded and played back using a terminal network of size $4 \times 4$. The engineered signals are used by these techniques for testing the sensitivity of nonlinearity modifications, which are then applied to real signals. These results show that nonlinear techniques are commonly used to detect pregnancy fluctuations and to reduce symptoms. 


\section{PROPOSED SYSTEM}

Fig. 3 shows an overview of the proposed structure. The flow of the methodology is depicted as follows: initially, the IoT devices are used to generate the data and then transmitted to the emergency subsystem for identifying any distress of FHS. The medical staff gets informed if any emergency is obtained. After all this, data is transferred to the cloud, where the calculation of features is processed and a prediction system based on one-dimensional DCGAN is used. At last, medical diagnoses are supported by this classification model and the results are provided to the clinical staff members for further analysis.

The medical staff only has access to patients' details and their diagnostic outcomes, where authentication, access control layer, and data encryption levels are used to provide communication protection for data privacy and confidentiality.

There are four parts in the developed solution, which are presented in Fig. 2. The integration of medical devices and sensors for the collection of data is presented in the first layer called the IoT layer, where the emergency subsystem is presented in the second layer (i.e. Fog computing layer) that is according to the inference rules and fixed clinical thresholds. The third and final part is considered as the Cloud computing layer, where signal processing techniques are used to extract the features in the third part, and classification of MFH is carried out by one-dimensional DCGAN in the fourth layer.

\section{A. IoT Layer}

Sensor and Equipment Devices which are used to collect medial parameters from mother and fetal.

The components that are presented in this layer are described as: Four vital signs of maternal such as blood pressure, temperature, heart rate of both Mother and fetal and oxygen saturation. Fetal and maternal monitoring are developed by using IoT modules, where diagnostic support is also provided by non-structured data.

To continuously monitor the fetus, the heart rate of his/her is only considered, which is obtained by the sensor of Doppler with a $4 \mathrm{~Hz}$ sampling rate. Then, a toco-dynamometer sensor is used to monitor the uterine tonus activity of maternal with the same sampling rate. Fig. 4 shows the modelling of the IoT interface layer.

At a $1 \mathrm{~Hz}$ sampling rate, important signals of maternal are obtained, where a photoplethysmography sensor is used to derive the oximetry and heart rate of maternal; then digital sensor integrated with medical devices is used to acquire the blood pressure for systolic and diastolic with temperature. Then, relevant events, audio, and photo notes of maternal that are acquired by medical staff are shared with the specialists, who are added to this system.

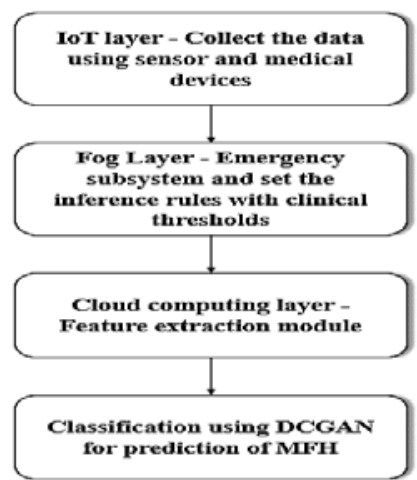

Fig. 2. The Flow of the Proposed Structure.

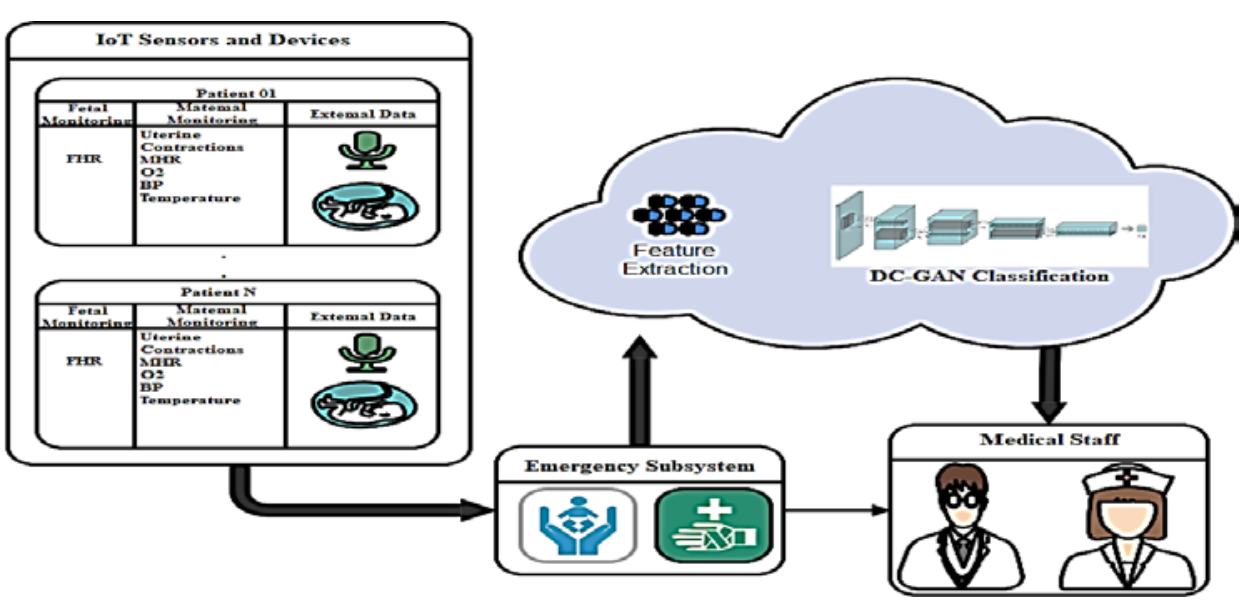

Fig. 3. Proposed Healthcare-IoT for Simultaneous Monitoring of a Mother and Fetus.

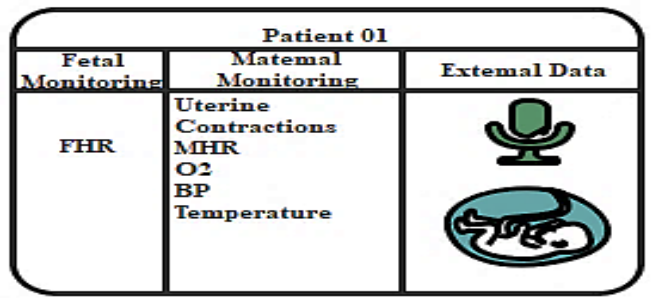

Fig. 4. Representation of Patient's Data with the Generation of non-external Data. 


\section{B. Fog Computing Layer: Subsystem for Emergency}

With limited resources of technology in remote clinical facilities and developing countries for providing ambulatory support, management and adoption of a cloud computing system for monitoring the real-time data is a challenging task. Therefore, architecture on fog computing is developed for supporting the requirements by creating a layer to collect the data on clinical premises.

For the signals of UC and heart rate of fetal, this type of external sensor is ideal for sound generation depending on the situation or the mother's movements [29]. Essentially, a preprocessor is included in the model before considering the specific dimensions of noise filtering and zero detection. The selected layer develops an automated analytical system for clinically important signals, according to the set of defined limits. The system is designed using three types of diagnostics (i.e. Emergency Class as EC) given in Table I.

According to the International Guidelines for Obstetricians and Gynaecologists (IGOG) [30], detailed descriptions of each standard portal and related classifications are provided in Table II for guidance on clinical group definitions and parental criteria.

\section{Cloud Computing Layer: Subsystem for Extraction of Automatic Features}

A data analytical module is created in this part for the extraction of maternal and fetus' features. To determine the parameters of UC and heart rate of fetus, signal processing techniques [31] are used and then non-linear and statistical metrics are calculated. The changes include long decelerations and baseline adjustments that need time to confirm and longterm analysis are required by interpretation of fetal's heart rate. Therefore, once the signal acquisition is done, after 10 minutes only the measures are calculated at the initial step and updated every 5 minutes for the entire examination.

There are two steps involved in this module, the initial importance is given for monitoring the fetal and finally, the vital signs of the mother has considered as second steps. For continuous monitoring, maximum information is achieved by analyzing the uterine contractions and heart rate of fetal by using signal processing techniques. In this sub-system, a total of 15 features are extracted for the analysis of the heart rate of the fetus that is established by medical experts, where the features include baseline value, baseline changes for instant and numbers, minima number, FHR minima instants, peaks for number and instants, decelerations of DIP-I and DIP-II, UC occurrences for number and instants, sample entropy for 5 and 20 minutes window, long decelerations and variable decelerations.

\section{Predictive Subsystem using DC-GAN Model}

GANs (Generative Adversarial Networks) are made up of two models that are trained concurrently using an adversarial approach. As shown in Fig. 5(a) and Fig. 5(b), a generator learns to make realistic images, whereas a discriminator learns to distinguish between actual and fraudulent images.

It should be noted that a total of 6 classes are considered and included in Table III for maternal and fetal diagnostic diagnoses based on medical group classification. In this work, a DC-GAN is used for the prediction process.

In this paper, the conditional DC-GAN is used to generate conditional deep changes according to DC-GAN [32] and cGAN [33]. In the original DC-GAN architecture, the bias was made up of step confusion layers, modular correction layers, and LeakyReLU activations.

The generator contains spasms, translucency, patches, and ReLU activations. From part 3 of the cloud computing layer, the input of the selected classifier consists of 15 features of the system to predict the six possible outputs, which are described in Table III. Fig. 5 shows the configuration of the DC-GAN state.

Instead of the original layer of convolutional-transpose, an up-sample and convolutional layer are used in a generator for avoiding the artifacts of checkerboard [34].If standard deconvolution is used to scale up from low to high precision, it uses all of the small feature points to 'plot' a larger square. This is where the "unequal overlap" problem comes in, i.e. when these "squares" converge on the larger side. In particular, D convolutions are random nests when the size of the kernel is not divisible by a step. Overlapping pixels create an unnecessary chessboard shape on the generated images. So, to get the "de-Convolution" function, we used the sample layer above instead. We scaled the feature using the top sample layer using billionaire (or adjacent interpolation) and then the convolutional layer. To achieve better results, a RelectionPad2d layer with size 1 is added before the convolutional layer for avoiding the boundary artifacts.

TABLE I. EMERGENCY SUBSYSTEM OUTPUT’S ClASSES

\begin{tabular}{|l|l|}
\hline Layer's Outcome & Explanation \\
\hline EC1 as 1 & Fetal Emergency (FE) \\
\hline EC2 as 2 & Maternal Emergency (ME) \\
\hline EC3 as 3 & Maternal and Fetal Emergency (FME) \\
\hline
\end{tabular}

TABLE II. LIST OF EMERGENCY LIMITS/THRESHOLDS BASED ON THE ABOVE GUIDELINES

\begin{tabular}{|l|l|l|l|}
\hline $\begin{array}{l}\text { Clinical } \\
\text { Parameter }\end{array}$ & Threshold & Interpretation & $\begin{array}{l}\text { Output } \\
\text { Class }\end{array}$ \\
\hline Heart Rate of Fetus & $>160$ & Normal tachycardia & 1 \\
\hline & $>180$ & Severe tachycardia & 1 \\
\hline & $<100$ & Normal bradycardia & 1 \\
\hline $\begin{array}{l}\text { Heart Rate of } \\
\text { Mother }\end{array}$ & $>100$ & Severe bradycardia & 1 \\
\hline $\begin{array}{l}\text { Tachycardia } \\
\text { Systolic blood } \\
\text { pressure }\end{array}$ & $>60$ & Bradycardia & 2 \\
\hline $\begin{array}{l}\text { Diastolic blood } \\
\text { pressure }\end{array}$ & $>90$ & Mother's hypoxemia & 2 \\
\hline Oximeter & $>37.5$ & Fever for mother & 2 \\
\hline Temperature & $\begin{array}{l}\text { Blood pressure for } \\
\text { mother }\end{array}$ & 2 \\
\hline
\end{tabular}


TABLE III. PREDICTED Classes FOR THE PROPOSED SUBSySTEM

\begin{tabular}{|l|l|}
\hline Output & Description \\
\hline PC1 & Normal for both Maternal and Fetal \\
\hline PC2 & Suspicious only on fetal \\
\hline PC3 & Distress on fetal \\
\hline PC4 & Suspicious only on maternal \\
\hline PC5 & Harmful for Maternal \\
\hline PC6 & Harmful for both \\
\hline
\end{tabular}

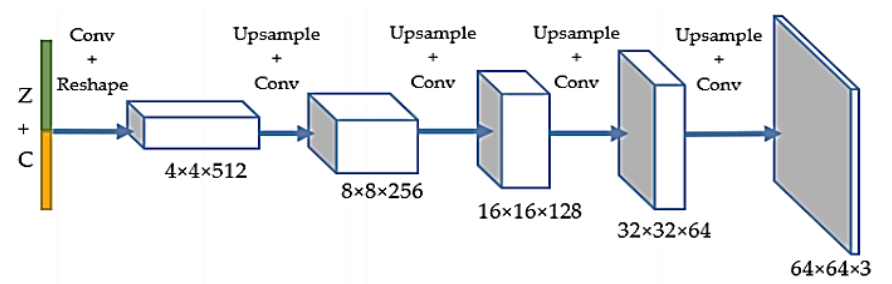

(a).Ḡenerator.

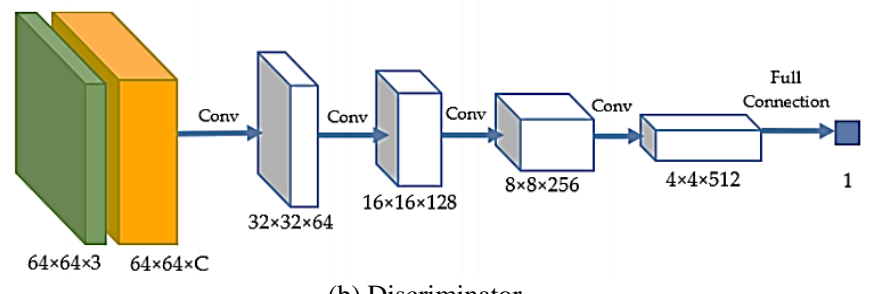

(b).Discriminator.

Fig. 5. Structure of Conditional DC-GAN.

To control the output function, the input model gets a class designation for the generator and the amplifier. The generator receives a random beep $(1 \times 1 \times \mathrm{Z})$ and a heat conditional sign $(1 \times 1 \times C)$, where the dimensions $\mathrm{Z}$ and $\mathrm{C}$ represent the number of categories. By default, we chose $\mathrm{Z}=100$ and $\mathrm{C}=$ 60 . They were combined and sent to the curved layer using a 1 $\times 1$ core. Output channel $8192(=4 \times 4 \times 512)$. Later, the output data was converted to $4 \times 4 \times 512$ format. Finally, we sent the data to several modules (except for Up-sample + Conv2D + Batch-Norm, the output layer) as high as the original DCGAN. An equal-valued data is up-sampled, only when the data size is $1 \times 1$, and therefore, input data i.e. concatenation of onehot label and random noise is not resized directly. Hence, the input noise size is kept at $1 \times 1 \times \mathrm{Z}$. To make the resizing step work, an extra convolutional layer is added in this model, where the $\mathrm{C}$ channel is encoded with a label in the discriminator. More precisely, the function label $\mathrm{n}$ is the data of the $\mathrm{H} \times \mathrm{W} \times \mathrm{C}$ scale, each $1 \mathrm{~s}$ and $0 . \mathrm{s}$ in the $\mathrm{n}$ channel. In the input layer, the feature is associated with the label: each feature is associated with the hotel. Channel direction label. Thus, the input channel size was $3+\mathrm{C}$. Similar to the original DC-GAN configuration, the Discriminator configuration has multiple configuration layers and a default volume layer (except for the output layer).

\section{E. Training Process and Loss Function}

We used the standard loss function for GANs, which is defined in [35] as follows: $\min _{G} \max _{D} V(D, G)=$

$\mathbb{E}_{x \sim p_{\text {data }}}(x)[\log D(x)]+\mathbb{E}_{x \sim p_{z}}(z)[\log (1-D(G(x))]$

There are two losses such as Loss_D andLoss_G used since a generator and a discriminator are presented in the GAN model. Here, the loss function is used as Binary CrossEntropy (BCE).

A sum of errD_real $=-\log (D(x))$ anderrD_fake $=$ $-\log (1-D(G(z)))$ is used to calculate the first loss, where the BCE is represented to the reality only when the fake inputs and real inputs are received by $\mathrm{D}$. The prediction of false positive is carried out by calculating the BCE when the fake inputs are received by D, i.e.Loss_G $=-\log (D(G(z)))$.

Set the default value for Adam optimizer as learning rate $=$ 0.0001 , when the training and updating of the generator and discriminator are carried out one by one. Finally, six possible outcomes are derived and the next section will show the validation of the proposed model with existing systems.

\section{VALIDATION AND RESUltS}

In this section, details of the datasets used in testing evaluation for a particular system are provided. Presenting the results obtained with the contingency subsystem, the following section validates the predictive subsystem feature of DCGAN with or without extrusion, using existing deep learning techniques. The performance measures used in this study are described in this section.

\section{A. Dataset Description}

Simulation experiments were performed on a standard antenatal CTG database from the UCI ML Repository (University of California, Irvine) [36]. CTG data were collected from 29 to 42 weeks gestation at SisPorto 2.0 in Portugal [37]. The database contains 2126 cases with 21 traits and cases classified according to FIGO guidelines by the consensus of three experts: 1655 general cases, 295 suspicious, and 176 pathological data.

\section{B. Performances Metrics}

In this section, major parameter metrics are discussed to validate the system model's performance. The correct identification of data samples over a total number of instances is calculated by using accuracy. An identification of MFS is often accurate by using the parameter called precision or positive predictive value. The possible outcomes are correctly classified by the proposed model is known as recall/sensitivity. The proportion of actual negatives is calculated by using the true negative rate/specificity. The harmonic average of sensitivity and precision is provided by F1-score or F-measure.

Accuracy is calculated as follows:

Accuracy $=\frac{T P+T N}{T P+T N+F P+F N}$

Precision is working out as following

Precision $=\frac{T P}{T P+F P}$ 
Sensitivity is deliberate as follows.

Recall $=\frac{T P}{T P+F N}$

Specificity and F-measure are defined as follows:

Specificity $=T N /(F P+T N)$ or $(1-F P R)$

$F 1-$ score $=2 T P /(2 T N+F P+F N)$

\section{Evaluation of Proposed Emergency Subsystem}

All emergencies have been classified by the organization. The results in Table IV show that the sensitivities (Caesarean deliveries as CI) for all classifiers are very low and the associated features are high. This is expected because the database is skewed in favor of genital records. Sensitivity and 95\% adjusted specificity for AUC and MSE are determined using the recommended CI model.

Disagreements during the modelling phase may result in poor performance. The integrating parameters from Table II are used to add the inference rule set to the system for improving the accuracy levels. For instance, the emergency of MFH is highly calculated by diagnosing the elevated blood pressure for systolic and diastolic, the temperature of maternal, etc. After discussing with the medical team, a total of twenty-six hypothetical rules are generated and here, five samples are provided in Table V.

The performance of the emergency subsystem based on the set of standard limits is shown in the Table VI.

It is important to note that performance is unsatisfactory with FP and FN rates high and sensitivities below $80 \%$, which is unacceptable given the critical interpretation of the contingency. The new set of proposed default rules has slightly improved the performance of the contingency.

TABLE IV. PERFORMANCE OF THE EMERGENCY SUBSYSTEM

\begin{tabular}{|l|l|l|l|l|}
\hline \multirow{2}{*}{$\begin{array}{l}\text { Outpu } \\
\text { t }\end{array}$} & \multicolumn{4}{|l|}{ Performance of Model before setting the static threshold } \\
\cline { 2 - 5 } & Sensitivity & Specificity & AUC & MSE \\
\hline \multirow{2}{*}{ ME } & $0.00(0.00,0.0$ & $0.99(0.99,0.9$ & $0.60(0.58,0.6$ & $0.08(0.07,0.0$ \\
& $0)$ & $9)$ & $1)$ & $8)$ \\
\hline \multirow{2}{*}{ FE } & $0.02(0.01,0.0$ & $0.99(0.99,0.9$ & $0.68(0.65$, & $0.08(0.07,0.0$ \\
& $3)$ & $9)$ & $0.69)$ & $8)$ \\
\hline \multirow{2}{*}{ FME } & $0.02(0.00,0.0$ & $0.99(0.99,0.9$ & $0.71(0.68,0.7$ & $0.08(0.07$, \\
& $4)$ & $9)$ & $3)$ & $0.08)$ \\
\hline
\end{tabular}

TABLE V. THE SUbCOMMITTEE ON CONCEPTUAL LAWS CONSIDERED TO DESCRIBE THE EMERGENCY FOR MOTHER OR FETUS

\begin{tabular}{|l|l|}
\hline Rule & Output \\
\hline$[$ MHR $>$ 90] and [blood pressure for systolic $>120]$ & ME \\
\hline $\begin{array}{l}[\text { Temperature }<36] \text { and [blood pressure for systolic }<100] \text { and } \\
{[\text { blood pressure for diastolic }<60]}\end{array}$ & ME \\
\hline$[$ MHR $>$ 80] and [FHR $>140]$ & FE \\
\hline$[$ FHR $>150]$ and [MT $>$ 37] & FE \\
\hline $\begin{array}{l}{[\text { blood pressure for diastolic }>13] \text { and [blood pressure for }} \\
\text { systolic }>13] \text { and [MHR }>\text { 90] and [FHR }>120]\end{array}$ & MFE \\
\hline
\end{tabular}

TABLE VI. EMERGENCY SUBSYSTEM PERFORMANCE UNDER EXPERT SUPERVISION AND DEFAULT RULES SET WITH FIXED LIMITS

\begin{tabular}{|c|c|c|c|c|}
\hline \multirow{2}{*}{$\begin{array}{l}\text { Outpu } \\
\mathrm{t}\end{array}$} & \multicolumn{4}{|c|}{ Considering the set of static thresholding } \\
\hline & Sensitivity & Specificity & AUC & MSE \\
\hline ME & $\begin{array}{l}0.76(0.70,0.81 \\
\text { ) }\end{array}$ & $0.56(0.54,0.58$ & $0.70(0.68,0.73$ & $0.08(0.07,0.08$ \\
\hline FE & $\begin{array}{l}0.71(0.68,0.73 \\
)\end{array}$ & $\begin{array}{l}0.82 \\
(0.80,0.85)\end{array}$ & $\begin{array}{l}0.87(0.86,0.88 \\
)\end{array}$ & $0.18(0.17,0.19$ \\
\hline FME & $\begin{array}{l}0.87(0.85,0.88 \\
)\end{array}$ & $\begin{array}{l}0.91 \\
(0.89,0.92)\end{array}$ & $\begin{array}{l}0.96(0.96,0.97 \\
)\end{array}$ & $0.08(0.07,0.09$ \\
\hline
\end{tabular}

The new set of proposed default rules has slightly improved the performance of the contingency subsystem. By considering only the maternal prognosis, the AUC (ME) is $70 \%$. For fetal emergency diagnosis, the system performance is AUC (FE) 87\%. This can happen because there is only one parameter that is directly related to the condition of the fetus, which is the FHR. Finally, in the worst-case scenario, when the mother and fetus have an emergency, the specific regimen performs best, with an AUC (FME) of 96\%.

\section{Performance Analysis of DC-GAN Prediction Subsystem}

In this section, five parameters are used to validate the proposed DC-GAN with existing techniques by considering with and without feature extraction techniques. and linear features. The existing technique called Convolutional Neural Network (CNN) [38] is designed for a smart healthcare system for monitoring the MFH. Multiple features extraction techniques are used to calculate both non-linear and non-linear features. The CNN network consists of six CLs, max-pooling, single flatten layer and output layer for the prediction process. The work [38] uses homogenous i.e. having the same neuron type in the entire network and based solely on the linearneuron model and didn't focus on non-linear features for better prediction. The data collected from 45 patients before labor and 55 patients during labor and validated the performance of CNN with existing ML techniques. But the results are not satisfactory, while using one-dimensional $\mathrm{CNN}$, which is advanced network called DC-GAN is incorporated with a smart healthcare system for monitoring the MFH. In addition, this proposed model uses the standard datasets for the prediction process and compared them with existing techniques such as CNN [38], Long-Short term memory (LSTM), etc.

1) Validation analysis of the proposed model without feature extraction: Initially, the experimental results of DCGAN are compared with CNN, LSTM, RNN, and DNN in terms of sensitivity, specificity, and precision, which are shown in Table VII and Fig. 6.

The proposed DCGAN model achieved $62.32 \%$ of precision and $77 \%$ of sensitivity and specificity. But, the DNN and LSTM model achieved nearly $46 \%$ to $50 \%$ of sensitivity, specificity, and precision. The reason is that the LSTM model requires more memory and takes a long time for training and it is easy to overfit. The DNN is also extremely expensive to train due to complex data models and it requires expensive GPUs. The CNN and RNN models achieved nearly 51\% to $52 \%$ of sensitivity, specificity, and the precision. 
TABLE VII. COMPARATIVE ANALYSIS OF VARIOUS DL ALGORITHMS WITHOUT FEATURE EXTRACTION

\begin{tabular}{|l|l|l|l|}
\hline \multirow{2}{*}{ DL Algorithms } & \multicolumn{3}{|l|}{ Parameter Metrics } \\
\cline { 2 - 4 } & Sensitivity & Specificity & Precision \\
\hline DC-GAN & 77.66 & 77.66 & 62.32 \\
\hline CNN & 52.27 & 52.27 & 51.65 \\
\hline RNN & 51.52 & 51.52 & 50.73 \\
\hline LSTM & 50.87 & 50.52 & 48.56 \\
\hline DNN & 47.85 & 48.65 & 46.5 \\
\hline
\end{tabular}

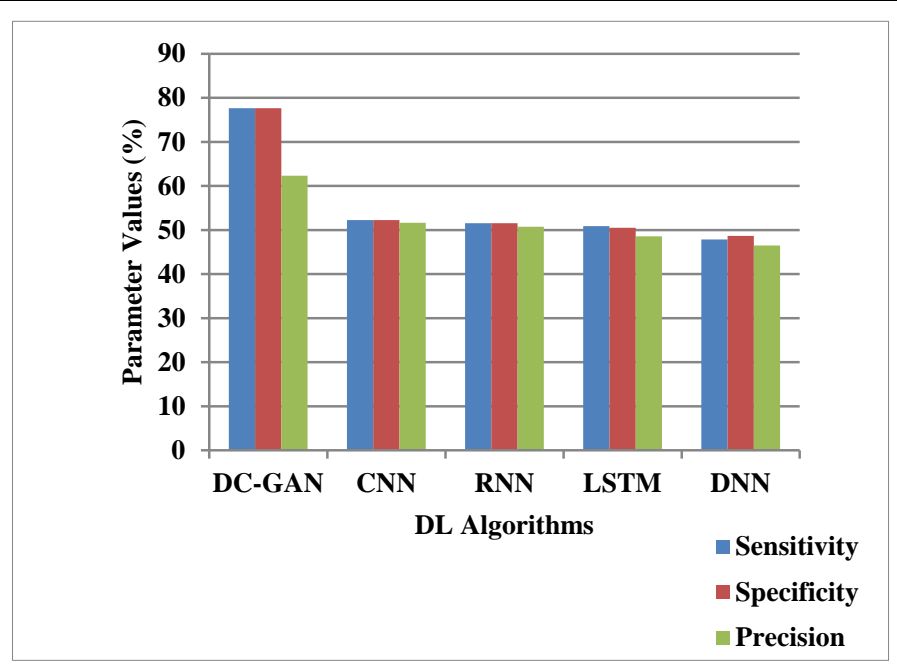

Fig. 6. Graphical Representation of Proposed DCGAN without Feature Extraction.

The CNN model requires more training data for accurate results, but now it has only three inputs of the emergency subsystem and provides low performance. The complex training procedures are presented in the RNN model and hence it achieved less than $60 \%$ of all parameters. Table VIII and Fig. 7 show the performance analysis of DCGAN and existing DL techniques in terms of accuracy and F1-score by implementing without feature extraction techniques.

In the accuracy analysis, DNN and LSTM achieved less performance (only 74\%) than other DL techniques, and proposed DCGAN achieved high performance (i.e. 89.03\%) than CNN and RNN techniques. But, the same proposed method achieved very low performance in terms of F1-score (i.e. 65.92\%), because the three inputs of the emergencysubsystem are directly given to the DCGAN model and it provides low performance since it is a one-dimensional model. Even though the proposed model achieved less performance, it provides better performance than CNN, RNN, LSTM, and DNN. The reason is that these existing techniques are unable to handle the inputs of the emergency subsystem due to its structures. DNN and LSTM model achieved nearly $46 \%$ to $49 \%$ of F1-score, whereas RNN and CNN achieved nearly $50 \%$ of F1-score.

2) Validation analysis of the proposed model with feature extraction: In this experimental analysis, the importance of feature extraction is revealed by implementing it with all the existing DL and proposed DCGAN models in terms of various parameters. Table IX and Fig. 8 show the performance analysis of different DL techniques in terms of specificity, precision, and sensitivity.

TABLE VIII. COMPARATIVE ANALYSIS OF VARIOUS DL ALgORITHMS WITHOUT FEATURE EXTRACTION

\begin{tabular}{|l|l|l|}
\hline DL Algorithms & Accuracy & F1-Score \\
\hline DC-GAN & 89.03 & 65.92 \\
\hline CNN & 81.66 & 51.72 \\
\hline RNN & 83.63 & 50.24 \\
\hline LSTM & 75.95 & 49.85 \\
\hline DNN & 73.54 & 46.21 \\
\hline
\end{tabular}

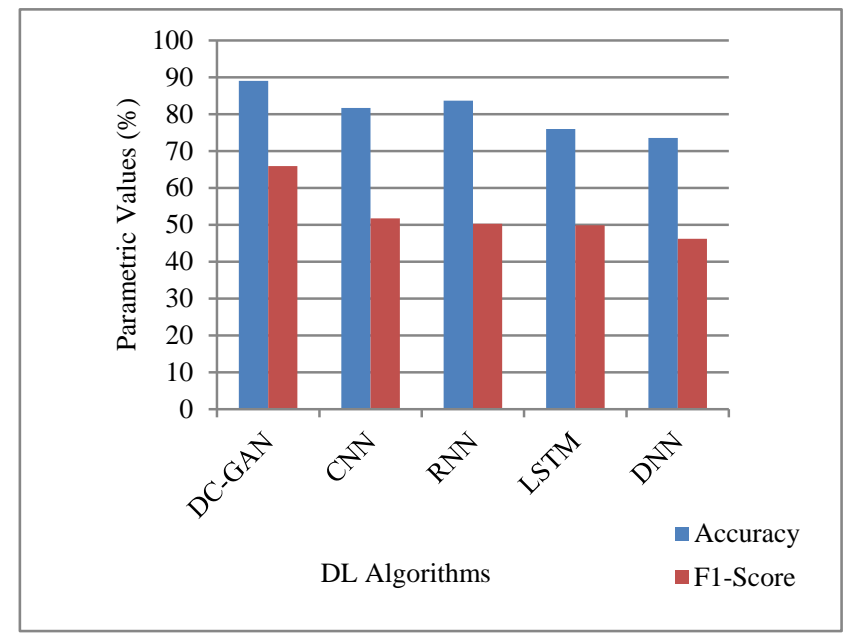

Fig. 7. Graphical Representation of Proposed DCGAN in terms of Accuracy and F1-score by considering without Feature Extraction.

TABLE IX. COMPARATIVE ANALYSIS OF VARIOUS DL ALGORITHMS WITH FEATURE EXTRACTION

\begin{tabular}{|l|l|l|l|}
\hline DL Algorithms & Sensitivity & Specificity & Precision \\
\hline DC-GAN & 98.27 & 96.70 & 97 \\
\hline CNN & 95.95 & 92.63 & 93 \\
\hline RNN & 97.11 & 94.62 & 95 \\
\hline LSTM & 91.62 & 84.57 & 85 \\
\hline DNN & 90.87 & 81.23 & 83 \\
\hline
\end{tabular}

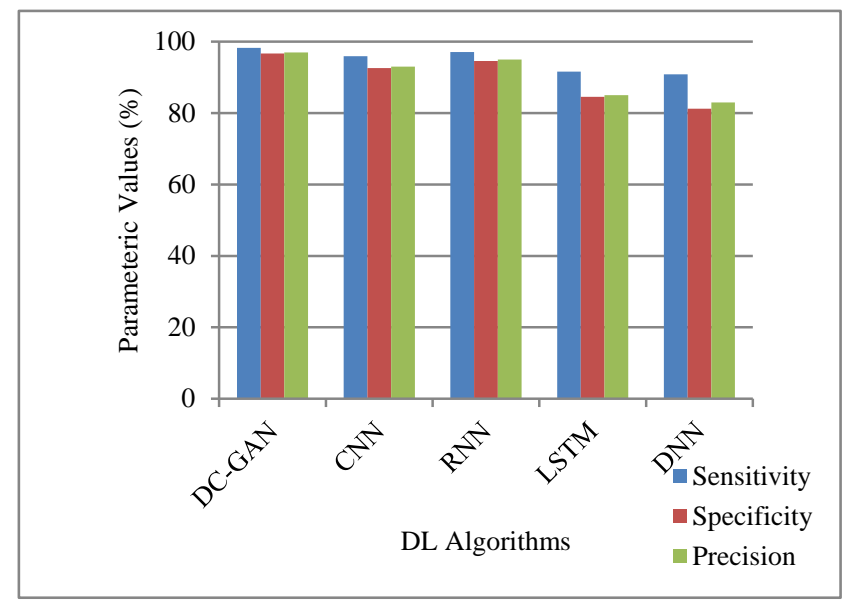

Fig. 8. Graphical Representation of Proposed DCGAN with Feature Extraction. 
When all the DL techniques are implemented with feature extraction, its performance is highly improved in terms of precision, specificity, and precision, while comparing it without feature extractions. This shows that feature extraction plays a major role in this study and the one-dimensional CNN, DCGAN, RNN, and DNN are used here and it requires feature extraction techniques for better performance. In the analysis of sensitivity, every DL technique achieved more than $90 \%$ and the proposed DCGAN achieved $98.27 \%$. The specificity of DCGAN is less (i.e. 96.70\%) than the precision (i.e.97\%) of proposed DCGAN, but its performance is highly improved while compared with existing DL techniques such as CNN, RNN, LSTM, and DNN. The CNN and RNN achieved nearly $92 \%$ to $95 \%$ of specificity and precision, whereas LSTM and DNN achieved nearly $81 \%$ to $85 \%$ of specificity and precision. This analysis shows that the proposed DCGAN model achieved better performance than other existing techniques. Table $\mathrm{X}$ and Fig. 9 show the experimental analysis of various DL algorithms in terms of accuracy and F1-score by considering feature extraction techniques.

TABLE X. COMParative ANALYSIS OF VARIOUS DL ALgORIthMS WITH FEATURE EXTRACTION

\begin{tabular}{|l|l|l|}
\hline DL Algorithms & Accuracy & F1-Score \\
\hline DC-GAN & 97.50 & 96 \\
\hline CNN & 96.03 & 94 \\
\hline RNN & 96.78 & 95 \\
\hline LSTM & 89.98 & 86 \\
\hline DNN & 88.58 & 84 \\
\hline
\end{tabular}

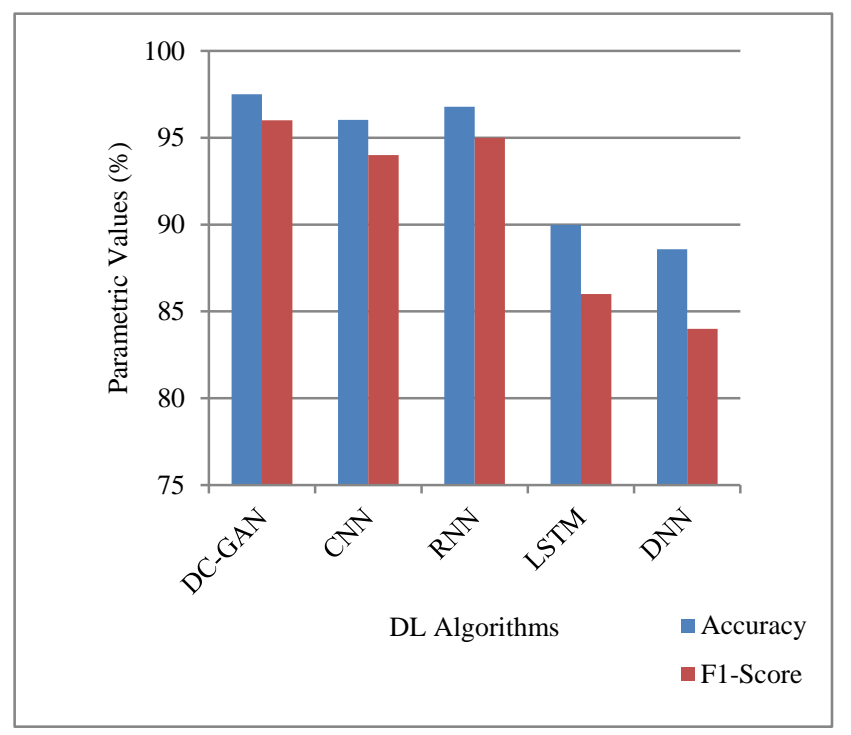

Fig. 9. Graphical Representation of Proposed DCGAN in Terms of Accuracy and F1-score by considering with Feature Extraction.

In the analysis of the F1-score, the LSTM and DNN achieved nearly $85 \%$, whereas CNN and RNN achieved nearly $94 \%$ to $95 \%$, but the proposed DCGAN achieved $96 \%$. The reason for the better performance of the proposed model is to introduce the conditional-based network in the DC-GAN, where the other techniques use only its basic architecture. In the experiments of accuracy, the DNN and LSTM techniques achieved 89\%, the CNN and RNN achieved nearly 96\%, and the proposed DCGAN network achieved $97.50 \%$ of accuracy while implementing feature extraction. Fig. 10 shows the comparative analysis of various DL algorithms in terms of accuracy by considering with and without feature extraction techniques for better validation purposes.

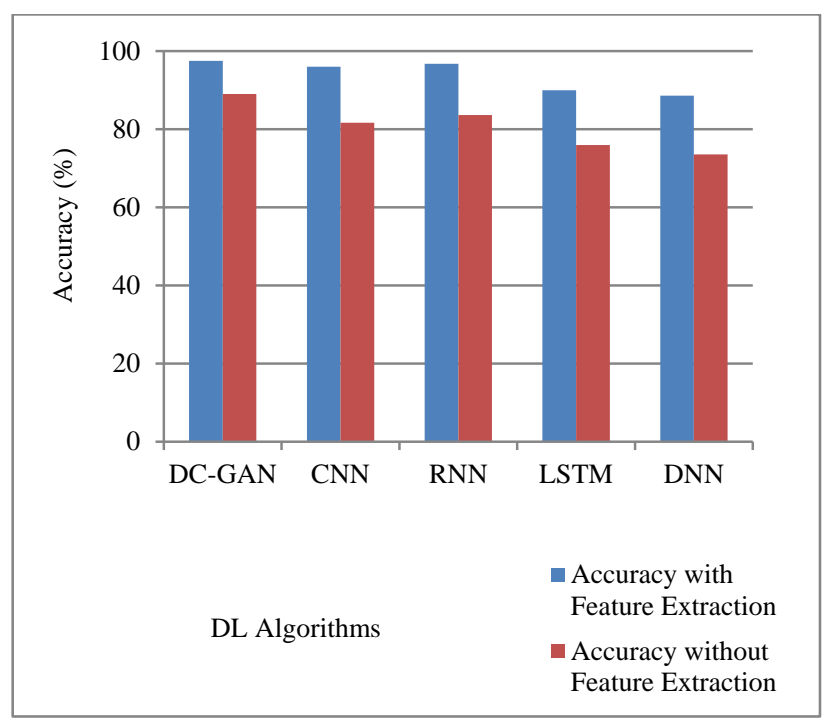

Fig. 10. Comparative Analysis of DL Techniques with and without Feature Extraction Techniques in Terms of Accuracy.

\section{CONCLUSION}

According to the diagnostic system's fog computing framework, an integrated solution is provided for high-risk monitoring of maternal patients, where this process relies on IoT-network-based sensors. A large data-analysis module for feature extraction techniques are used to extract features from the observed signals, and finally, an in-depth DCGAN-based classification system for predicting maternal-fetal health by the possible outcomes as PC1 to PC6 for performance analysis. The main contribution of this work in the Emergency Diagnostics Branch is the design of a set of hypothetical rules that can achieve more than $80 \%$ of the area under the curve for fetal, maternal, and emergencies. A total of 6 possible outcomes from the prediction model were considered. Compared with existing DL techniques such as CNN, LSDM, RNN, and DNN, the results show that DCGAN techniques and homogeneous systemic extraction performed better in the maternal and fetal and stage II. In the future, the efficiency of the emergency diagnostic system can be improved by modifying the selected model using different hypothetical rules.

\section{REFERENCES}

[1] WHO. Maternal mortality, Retrieved on 2019. http://www.who.int/ mediacentre/factsheets/fs348/en/.

[2] WHO. Aging and health, Retrieved on 2019. http://www.who.int/ mediacentre/factsheets/fs404/en/.

[3] L. Atzori, et al., The internet of things: a survey, Computer Network. 54 (15) (2010) 2787-2805.

[4] J.A. Stankovic, et al., Research directions for the internet of things, IEEE Internet Things J. 1 (1) (2014) 3-9. 
[5] J. Gubbi, et al., Internet of things (IoT): A vision, architectural elements, and future directions, Future Generation Computer Sysem. 29 (7) (2013) 1645-1660.

[6] R. Mieronkoski, et al., The internet of things for basic nursing care-a scoping review, International Journalof Nursing Studies. 69 (2017) 7890.

[7] W. Gyselaers, V. Storms, L. Grieten, New technologies to reduce medicalization of prenatal care: a contradiction with realistic perspectives, Expert review of medical devices. 13 (8) (2016) 697-699,

[8] Y. Lu, X. Zhang, X. Fu, F. Chen, K.K.L. Wong, Ensemble machine learning for estimating fetal weight at varying gestational age, in Proceedings of the Thirty-Third AAAI Conference on Artificial Intelligence, AAAI 2019, 2019, pp. 9522-9527,

[9] J. Li, L. Huang, Z. Shen, Y. Zhang, M. Fang, B. Li, X. Fu, Q. Zhao, H. Wang, Automatic classification of fetal heart rate based on convolutional neural network, IEEE Internet Things J. 6 (2) (2019) 1394-1401.

[10] Y. Lu, X. Zhang, L. Jing, X. Li, X. Fu, Estimation of the fetal heart rate baseline based on singular spectrum analysis and empirical mode decomposition, Future Generation Computer System 112 (2020) 126135.

[11] Y. Lu, Y. Gao, Y. Xie, S. He, Computerised interpretation systems for cardiotocography for both home and hospital use, in: Proceedings of the 31st IEEE International Symposium on Computer-Based Medical Systems, CBMS 2018, IEEE, 2018, pp. 422-427,

[12] Y. Lu, Y. Qi, X. Fu, A framework for intelligent analysis of digital cardiotocographic signals from IoMT-based fetal monitoring, Future Gener. Comput. Syst. 101 (2019) 1130-1141,

[13] Lu, Y., Fu, X., Chen, F., \& Wong, K.K, "Prediction of fetal weight at varying gestational age in the absence of ultrasound examination using ensemble learning”, Artificial intelligence in medicine, 102, 101748 . (2020).

[14] C.Perera, C.H. Liu, S. Jayawardena, The emerging internet of things marketplace from an industrial perspective: A survey, IEEE Trans. Emerg. Top. Comput. 3 (4) (2015) 585-598,

[15] H. Ghasemzadeh, E. Guenterberg, R. Jafari, Energy-efficient information-driven coverage for physical movement monitoring in body sensor networks, IEEE Journal of. Selective. Areas Communication. 27 (1) (2009) 58-69,

[16] K. Wac, M.S. Bargh, B. jan F. Van Beijnum, R.G. Bults, P. Pawar, A. Peddemors, Power- and delay-awareness of health tele-monitoring services: the mobi-health system case study, IEEE Journal on Selected Areas in Communications. 27 (4) (2009) 525-536.

[17] G. Chiarini, P. Ray, S. Akter, C. Masella, A. Ganz, "mHealth technologies for chronic diseases and elders: A systematic review",IEEE Journal on Selected Areas in Communications. 31 (9) (2013) 6-18,

[18] Oh, J. Cha, M. Ji, H. Kang, S. Kim, E. Heo, J.S. Han, H. Kang, H. Chae, H. Hwang, S. Yoo, Architecture design of healthcare software-as-aservice platform for cloud-based clinical decision support service, Healthcare Inf. Res. 21 (2) (2015) 102-110,

[19] A.J. Jara, M.A. Zamora-Izquierdo, A.F.S. and, Interconnection framework for mhealth and remote monitoring based on the internet of things, IEEE Journal on Selected Areas in Communications. 31 (9) (2013) 47-65,

[20] M. Barcelo, A. Correa, J. Llorca, A.M. Tulino, J.L. Vicario, A. Morell, IoT-Cloud service optimization in next-generation smart environments, IEEE Journal on Selected Areas in Communications. 34 (12) (2016) 4077-4090,

[21] Y. Huang, M.Y. Hsieh, H.C. Chao, S.H. Hung, J.H. Park, "Pervasive, secure access to a hierarchical sensor-based healthcare monitoring architecture in wireless heterogeneous networks", IEEE Journal on Selected Areas in Communications. 27 (4) (2009) 400-411,
[22] D. Niyato, E. Hossain, S. Camorlinga, Remote patient monitoring service using heterogeneous wireless access networks: architecture and optimization, IEEE Journal on Selected Areas in Communications. 27 (4) (2009) 412-423,

[23] M.A. Salahuddin, A. Al-Fuqaha, M. Guizani, K. Shuaib, F. Sallabi, Softwarization of internet of things infrastructure for secure and smart healthcare, Computer 50 (7) (2017) 74-79,

[24] Allahem, Hisham and Srinivas Sampalli, "Automated uterine contractions pattern detection framework to monitor pregnant women with a high risk of premature labor", Informatics in Medicine Unlocked, vol.20, pp.100404, 2020.

[25] Liu L, Jiao Y, Li X, Ouyang Y, and Shi D, "Machine learning algorithms to predict early pregnancy loss after in vitro fertilizationembryo transfer with fetal heart rate as a strong predictor", Computer Methods and Programs in Biomedicine, vol.196, no.105624, 2020.

[26] Testa, A. and Jackson, D.B., 2020. Incarceration exposure and maternal food insecurity during pregnancy: Findings from the Pregnancy Risk Assessment Monitoring System (PRAMS), 2004-2015. Maternal and child health journal, 24(1), pp.54-61.

[27] Azimi I, Pahikkala T, Rahmani A. M, Niela-Vilén H, Axelin A, and Liljeberg P, "Missing data resilient decisionmaking for healthcare IoT through personalization: A case study on maternal health”, Future Generation Computer Systems, vol.96, pp.297-308, 2019.

[28] Mourad M, A. Diab, M. Khalil, and C. Marque, "Pregnancy/Labor Discrimination and Monitoring: An Investigation Using Nonlinear Methods", International Arab Conference on Information Technology (ACIT), Werdanye, Lebanon, pp.1-4, 2018.

[29] J. A. Lobo Marques, P. C. Cortez, J. P. D. V. Madeiro, S. J. Fong, F. S. Schlindwein, and V. H. C. D. Albuquerque, "Automatic Cardiotocography Diagnostic System Based on Hilbert Transform and Adaptive Threshold Technique," in IEEE Access, vol. 7, pp. 7308573094, 2019.

[30] Ayres-de-Campos, D., Spong, C.Y., \&Chandraharan, E. (2015). “FIGO consensus guidelines on intrapartum fetal monitoring: Cardiotocography”, International Journal of Gynecology\& Obstetrics, 131. 2015.

[31] Q. Zhang, L. T. Yang, Z. Chen, P. Li and F. Bu, "An Adaptive Dropout Deep Computation Model for Industrial IoT Big Data Learning With Crowdsourcing to Cloud Computing," in IEEE Transactions on Industrial Informatics, vol. 15, no. 4, pp. 2330-2337, April 2019.

[32] Radford, A.; Metz, L.; Chintala, S. Unsupervised representation learning with deep convolutional generative adversarial networks. arXiv 2016, arXiv:1511.06434.

[33] Mirza, M.; Osindero, S. Conditional generative adversarial nets. arXiv 2014, arXiv:1411.1784.

[34] Sugawara, Y., Shiota, S., \& Kiya, H. "Convolutional Neural Networks Without Any Checkerboard Artifacts”, 2018 26th European Signal Processing Conference (EUSIPCO), 1317-1321. 2018.

[35] Goodfellow, I.J.; Pouget-Abadie, J.; Mirza, M.; Xu, B.; Warde-Farley, D.; Ozair, S.; Courville, A.; Bengio, Y. Generative adversarial nets. In Advances in Neural Information Processing Systems 27; Ghahramani, Z., Welling, M., Cortes, C., Lawrence, N.D., Weinberger, K.Q., Eds.; Morgan Kaufmann Publishers Inc.: Burlington, MA, USA, 2014.

[36] A. Asuncion and D. J. Newman. "UCI machine learning repository,"[http://www.ics.uci.edu/ mlearn/MLReposit-ory.html] University of California, School of Information and Computer Science, Irvine, CA, 2007.

[37] Ayresde, C.D., Bernardes, J., Garrido, A., et al.: SisPorto 2.0: “A program for automated analysis of cardiotocograph", The Journal of Maternal-Fetal\& Neonatal Medicine. 9(5), 311-318 (2000).

[38] Marques, J.A.L., Han, T., Wu, W., do Vale Madeiro, J.P., Neto, A.V.L., Gravina, R., Fortino, G. and de Albuquerque, V.H.C., 2020. IoT-based Smart Health System for Ambulatory Maternal and Fetal Monitoring. IEEE Internet of Things Journal.,2020. 